\title{
Channel management for WATM mobile satellite systems
}

\begin{abstract}
This paper presents a dynamic channel allocation scheme for wireless asynchronous transfer mode (WATM) satellite systems. Handoff schemes of WATM are utilized to deal with handoff issues in a WATM mobile satellite system. Here we investigate and simulate a dynamic channel allocation scheme for handoff in WATM mobile satellite networks, which improves the network resource utilization, by measuring the network performance in terms of new call blocking probability (NCBP) and handoff call blocking probability (HCBP). Arriving calls are given channels based on their priority and calls are subdivided into real time and non real time calls. Highest priority is given to real time handoff calls, followed by non real time handoff calls, then real time new calls and finally non real time new calls.
\end{abstract}

Keyword: WATM mobile satellite system; Handoff call blocking; New call blocking probability; Channel management 